\title{
HEXAMMINE NICKEL COMPLEX: COOPERATIVE ROTATION OF SIX $\mathrm{NH}_{3}$ GROUPS
}

\author{
P.B. SCZANIECKI \\ Institute of Molecular Physics, Polish Academy of Sciences \\ Smoluchowskiego 17, 60-179 Poznań, Poland
}

(Received July 5, 1994)

\begin{abstract}
Electrostatic interactions between $\mathrm{NH}_{3}$ groups undergoing a correlated rotation are calculated with reference to the nickel hexammine complexes $\left[\mathrm{Ni}\left(\mathrm{NH}_{3}\right)_{6}\right]^{2+}$. The results demonstrate the angular dependence of the cis and trans arrangements of the model ammonia triangles. The previously defined four maxima and four minima are identified.
\end{abstract}

PACS numbers: $76.30 .-\mathrm{v}$

\section{Introduction}

Hexammine complexes of divalent metal ions exhibit the fluorite type of structure with the complex cations $\left[\mathrm{Me}\left(\mathrm{NH}_{3}\right)_{6}\right]^{2+}$ in the calcium sites in the face centered lattice [2]. Figure 1 shows one eighth of the elementary cell of the lattice

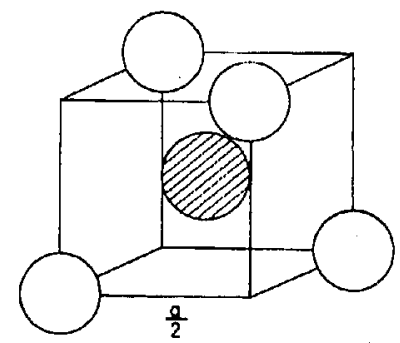

Fig. 1. One eighth of the $\left.\mathrm{Ni}\left(\mathrm{NH}_{3}\right)_{6}\right] \mathrm{Y}_{2}$ unit cell: the central cation (shadowed circle) is shown in its tetrahedral environment of $\mathrm{Y}$ anions. $a$ is the lattice constant.

constant $a$ for the compounds of the formula $\left[\mathrm{Me}\left(\mathrm{NH}_{3}\right)_{6}\right] \mathrm{X}_{2}$, where the central complex cation is tetrahedrally surrounded by the anions $\mathrm{X}$. These can either be the halides or complex anions like $\mathrm{NO}_{3}^{-}, \mathrm{BF}_{4}^{-}, \mathrm{ClO}_{4}^{-}$or $\mathrm{PF}_{6}^{-}$. 


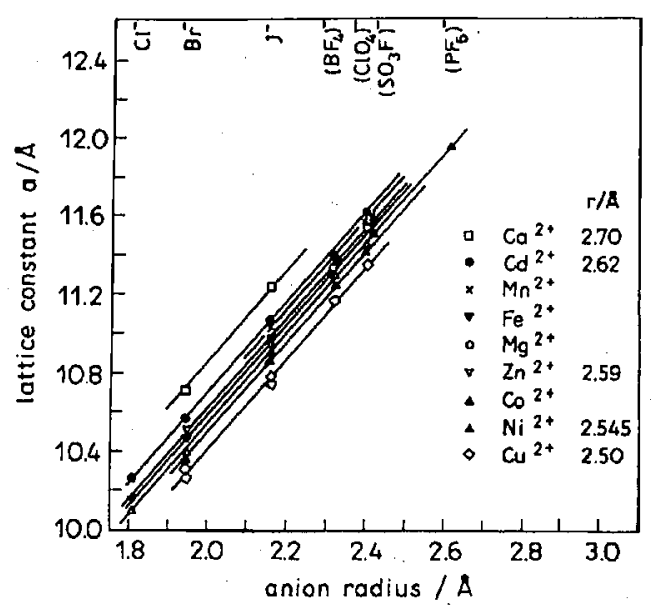

Fig. 2. Lattice constants $a$ and the ionic radii for the selection of hexammine salts. The solid lines correspond to the same metal ion.

The model based on close-packed spheres may be used for an estimation of the radii of the hexacoordinated metal cations $\left[\mathrm{Me}\left(\mathrm{NH}_{3}\right)_{6}\right]^{2+}$, with $\mathrm{Me}=\mathrm{Ni}^{2+}$, $\mathrm{Co}^{2+}, \mathrm{Zn}^{2+}, \mathrm{Cu}^{2+}$ and $\mathrm{Cd}^{2+}$. The diagrams shown in Fig. 2 display the data available $[2,3]$ for the group of typical hexammines: the lines joining the groups of the points obey the relation $a=\frac{4}{\sqrt{3}}\left(r+r_{a}\right)$, where $r$ and $r_{a}$ refer to ionic radii of the anions and cations, respectively. According to these diagrams, the radius of the complex cation may vary from $2.70 \AA$ for calcium to $2.55 \AA$ and $2.50 \AA$ for nickel and copper.

Anomalous dependence of some physical properties of the nickel hexammines on temperature and pressure [4-8] reflects the phase transformation occurring in these salts: from the regular, fluorite-type of structure above $T_{\mathrm{c}}$ to a lower symmetry at temperatures below $T_{\mathrm{c}}[9,10]$. Taking into account the differences in the stereochemistry of the anions it is possible to correlate the temperatures of the phase transformations with lattice parameters [11].

In electron paramagnetic resonance (EPR) the phase transition in nickel hexammines manifests itself through a linewidth broadening; it is reasonable to attribute this broadening to a symmetry correlated configuration of all 18 protons in the hexammine complex. Theoretical model has been proposed by Bates and Stevens [1] in the past, when the details of the structural transformations in nickel hexammine halides have not yet been available. The model is based on the calculation of the electrostatic energy of all the available configurations of the ammonia triangles in $\left[\mathrm{Ni}\left(\mathrm{NH}_{3}\right)_{6}\right]^{2+}$ complex and attempts to account for the observed entropy changes [1].

The present work gives the details of the calculation of the electrostatic energy of the model complex of six $\mathrm{NH}_{3}$ groups as a function of the angle $\alpha$ of correlated rotation of all the protons. 


\section{Model of electrostatic interactions in $\left[\mathrm{Ni}\left(\mathrm{NH}_{3}\right)_{6}\right]^{2+}$ complex}

The hexammine complex cation can be schematically visualized as a cube of $2 r$ edge (Fig. 3). For simplicity, in the calculation of the electrostatic energy of the six ammonia groups, the metal ion at $(0,0,0)$ coordinates in the center of the cube will be omitted. The coordinates of the protons indexed with $i=1,2,3$ in

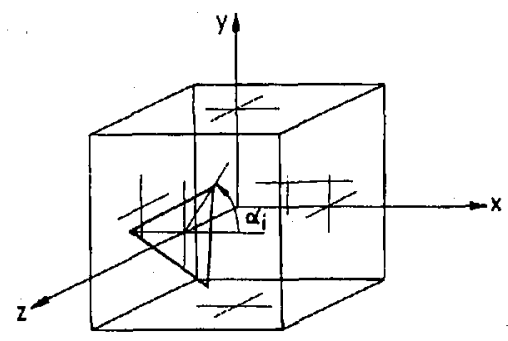

Fig. 3. The definition of $x, y, z$ coordinates used in the description of the rotation of ammonia triangles. The cube of the edge $2 r$ visualize the $\left[\mathrm{Ni}\left(\mathrm{NH}_{3}\right)_{6}\right]^{2+}$ complex. Only one of the six triangles has been drawn. $\alpha$ is the angle of its rotation in (001) plane.

the face (001) will be described with the vectors $\boldsymbol{R}_{i}=\left(\rho \cos \alpha_{i}, \rho \sin \alpha_{i}, r\right)$ where $\rho$ denotes $\mathrm{N}-\mathrm{H}$ distance and

$$
\alpha_{i}=\alpha+(i-1) \frac{2 \pi}{3} \text {. }
$$

The coordinates of the protons of the remaining $\mathrm{NH}_{3}$ groups are defined in similar way. For the pair of interacting $\mathrm{NH}_{3}$ groups the electrostatic energy of protons assigned an effective electric charge $\beta|e|$ ( $e$ - electron charge), whose coordinates are $\boldsymbol{R}_{i}$ and $\boldsymbol{R}_{j}$, is

$$
E=\sum_{i, j} \frac{\beta^{2} e^{2}}{\left|\boldsymbol{R}_{i}-\boldsymbol{R}_{j}\right|}, \quad i, j=1,2,3 .
$$

The contributions from all the $\mathrm{NH}_{3}$-pairs are accounted for those separated by $2 r$ and the near neighbors whose distance is $\sqrt{2} r$ according to the systematics given in Table. Symbols $X, Y, Z$ refer to the $\mathrm{NH}_{3}$ groups situated on the faces (100), (010) and (001) of the cube while the bar symbols $\bar{X}, \bar{Y}, \bar{Z}$ denote the positions of the ammonia triangles placed on the opposite faces (Fig. 3). As an example, the electrostatic energy of the three pairs of $\mathrm{NH}_{3}$ groups $X X, Y Y$ and $Z Z$ is given below in $\beta^{2} e^{2} / r$ units

$$
E^{++}=\frac{3}{\sqrt{2}} \sum_{i, j}\left[\left(1+\kappa^{2}\right)-\kappa \cos \alpha_{i}-\kappa \sin \alpha_{j}-\kappa^{2} \sin \alpha_{i} \cos \alpha_{j}\right],
$$

where $\kappa=\rho / r$ and $i, j=1,2,3$.

Similar formulae describe the electrostatic energy of $X Y, X Z$ and $Y Z$ pairs; the differences are in the relative signs of the components. The contribution due to $X \bar{X}, Y \bar{Y}, Z \bar{Z}$ pairs of $\mathrm{NH}_{3}$ groups does not depend on the rotation angle $\alpha$ and amounts to

$$
E^{0}=\frac{9}{2}+18 \sqrt{4+3 \kappa^{2}}
$$




$$
E^{0}=\frac{9}{2} \sqrt{1+\kappa^{2}}+18 \sqrt{4+\kappa^{2}},
$$

for cis and trans configuration, respectively.

The motion of all the ammonia triangles is assumed to be coordinated, hence the variation of the energy of the idealized hexammine complex can be displayed as a function of unique parameter $\alpha$. Substituting $\alpha_{i}=\alpha+(i-1) 120, \alpha_{j}=$ $\alpha+(j-1) 120$ and summing up for all the nine contributions of each of 15 pairs of the ammonia triangles (Table), one obtains the changes of the electrostatic energy of the idealized hexammine metal complex related to the rotation of the proton triangles by the angle $\alpha$ (Fig. 4). As a reference level the energy of the system in

\section{TABLE}

Systematics of the $\mathrm{NH}_{3}$ pairs in the metal hexamine octahedron.

\begin{tabular}{c|c|c|c|c|c|c}
\hline \hline & $X$ & $Y$ & $Z$ & $\bar{X}$ & $\bar{Y}$ & $\bar{Z}$ \\
\hline$X$ & & ++ & ++ & 0 & +- & -+ \\
$Y$ & & & ++ & -+ & 0 & +- \\
$Z$ & & & & +- & -+ & 0 \\
$\bar{X}$ & & & & & -- & -- \\
$\bar{Y}$ & & & & & & -- \\
$\bar{Z}$ & & & & & &
\end{tabular}

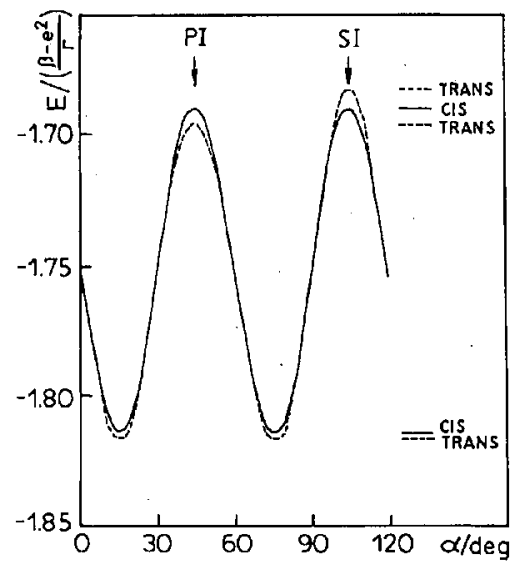

Fig. 4. Electrostatic energies of the six ammonia groups in the model of hexammine complex cation where $\kappa=0.4$ and $\alpha$ is the angle of simultaneous, coordinated rotation of all the $\mathrm{NH}_{3}$ groups preserving the trigonal symmetry along (111) direction of the complex. PI and SI refer to points-in and sides-in arrangements, respectively, as defined earlier [1]. 
which all the charges occupy the center of each triangle was adopted, i.e. $\rho=0$. It amounts to $27 / 2+54 \sqrt{2}$ in $\beta^{2} e^{2} / r$ units.

The plots in Fig. 4 describe the energies of trans and cis configuration of the idealized $\mathrm{NH}_{3}$, respectively. The angle $\alpha$ describes the simultaneous rotation of all the three pairs of charged triangles $X X, Y Y$ and $Z Z$ for which the trigonal symmetry along the body diagonal of the cube is maintained (Fig. 3). Center of inversion of the whole complex is present in the trans configuration.

\section{Discussion of the results}

Both trans and cis configuration exhibit two minimal energy arrangements of the triangles: at $\alpha=15$ and $\alpha=75$ degree. The trans configuration attains two distinctly different, maximum energy values at $\alpha=45$ and $\alpha=105$ degree; in the earlier paper Bates and Stevens, who found the values of the minimum and maximum energy of the hexammine cluster [1] refer to these configurations of $\mathrm{NH}_{3}$ triangles as points-in and sides-in arrangements, respectively. Similarly, the remaining configurations [1], can all be identified as four minima and four maxima shown in Fig. 4. Magnitude of the energy variations resulting from the correlated rotation of the ammonia groups can also be learned from these plots.

Substituting $\rho=1 \AA$ and $r=2.5 \AA$ for the size of $\mathrm{NH}_{3}$ and Ni-Ni distance, respectively and assigning $0.3|e|$ for the effective proton charge in each corner of the triangle, the energy barriers can be determined at each configuration. They are $550 \mathrm{~cm}^{-1}$ for trans and about $500 \mathrm{~cm}^{-1}$ for cis configuration whereas the differences at the positions of the minima in Fig. 4 are of the order of a few wave numbers.

The plots obtained under these very simplified assumptions illustrate how the correlated rotation of $\mathrm{NH}_{3}$ groups, while preserving the trigonal symmetry, may affect the energy of the complex. It should be realized, as indicated previously [1], that the transitions between the discrete minima and maxima (Fig. 4) do not have to traverse along the path of correlated motion of $\mathrm{NH}_{3}$ groups. However, the energies available to this electrostatic model of six ammonia groups coordinating metal ion, are all contained within the limits of the minimum and the maximum of the configuration trans.

For the interpretation of EPR lineshape it can be important that the minima of the trans and cis configuration are separated merely by a few wave numbers in energy scale (Fig. 4), hence, they are of the order of Zeeman splitting in EPR. Typical splittings of the electronic multiplets of $\mathrm{Ni}^{2+}$ in crystal field are $10^{4} \mathrm{~cm}^{-1}$ and the zero-field splitting in nickel hexammines is about $1 \mathrm{~cm}^{-1}$ [12-14].

Excluding the ammonia groups, the hexacoordinated nickel complex $\left[\mathrm{Ni}\left(\mathrm{NH}_{3}\right)_{6}\right]^{2+}$ was treated as rigid in the calculations; in the environment of a lower symmetry, which occurs in the low-temperature phase of the hexammine salts [15] this complex is expected to distort.

\section{Acknowledgments}

The author is very indebted to Professor Jan Stankowski for his valuable comments and discussions helpful in preparation of this manuscript. 


\section{References}

[1] A.R. Bates, K.W.H. Stevens, J. Phys. C 2, 1573 (1969).

[2] R.W.G. Wyckoff, Crystal Structures, Vol. 3, Interscience, New York 1965, p. 783.

[3] D’Ans Lax, Taschenbuch für Chemiker und Physiker, Springer Verlag, Berlin 1970.

[4] A. Elgsaeter, I. Svare, J. Phys. Chem. Solids 31, 1405 (1970).

[5] J. Stankowski, J.M. Janik, A. Dezor, B. Sczaniecki, Phys. Status Solidi A 16, K167 (1973).

[6] P.B. Sczaniecki, M. Grimshaw, W.S. Moore, Acta Phys. Pol. A 56, 503 (1979).

[7] M. Krupski, J. Stankowski, Phys. Status Solidi A 45, 585 (1978).

[8] M. Krupski, J. Stankowski, Acta Phys. Pol. A 55, 597 (1979).

[9] E. Dynowska, Phys. Status Solidi A 31, K23 (1975).

[10] S. Hodorowicz, M. Ciechanowicz-Rutkowska, J.M. Janik, J.A. Janik, Phys. Status Solidi A 43, 53 (1977).

[11] J. Stankowski, Mater. Sci. 2, 3 (1976).

[12] C. Trapp, Chin I-Shyr, J. Chem. Phys. 54, 196 (1971).

[13] P.B. Sczaniecki, J. Stankowski, Acta Phys. Pol. A 51, 117 (1977).

[14] J.A. Ochi, S. Isotani, W. Sano, J. Phys. Chem. Solids 43, 411 (1982).

[1.5] E. Dynowska, J. Stankowski, Phys. Status Solidi A 52, 381 (1979). 